\title{
Design of Rotary Root Stubble Digging Machine Based on Solidworks*
}

\author{
Xinglong Liao ${ }^{1}, \mathrm{Xu} \mathrm{Ma}^{1,2}$, and Yanjun Zuo ${ }^{1}$ \\ ${ }^{1}$ College of Engineering, South China Agricultural University, Guangzhou, P.R. China \\ ${ }^{2}$ Key Laboratory of Key Technology on Agricultural Machine and Equipment, \\ Ministry of Education, South China Agricultural University, Guangzhou, P.R. China \\ liaoxinglong1985@163.com, maxu1959@scau.edu.cn, 42648135@qq.com
}

\begin{abstract}
In the paper, the necessity of root stubble harvesting and recycling was put forward from the perspective of biomass energy utilization. To accomplish mechanized harvesting on root stubble, a rotary digging machine was designed based on parametric modeling software Solidworks. Firstly, parts were built under entity modeling module, and then assembled to 4 main mechanisms in assembling environment. Secondly, mechanisms including frame, transmission mechanism, suspension mechanism and digging mechanism were assembled together to establish the whole prototype on which interference checking was done. Through manual change of the transmission chain's installation position, the digging mechanism was able to shift between reverse and forward rotation according to different soil conditions. Finally, relevant 2-D engineering drawings were generated for manufacture. The paper provides methodological reference for the design of similar machines and preparation for further simulation and analysis of the designed models.
\end{abstract}

Keywords: Root stubble, Rotary digging machine, Solidworks, Virtual design.

\section{Introduction}

As one of the main food crops in China, corn's significance is only next to rice and wheat. The perennial planting area is about 25 million $\mathrm{hm} 2$ and annual yield is up to 120 million tons ${ }^{[1]}$. As by-product of corn planting, the treatment of these root stubble is a tough task to peasants, especially in busy farming seasons. Moreover, there exist many deficiencies in traditional treating ways of root stubble. Leaving them alone will hinder subsequent seeding operation; burning them up will generate a lot of smoke harmful to the environment; burying through plowing will be inefficient; and mechanized shattering will consume large amounts of energy. Stalk and root stubble of corn is a kind of clean fuel with high heating value and low sulfur and is one of the most potential green renewable energies. As energy crisis and environmental pollution are more and more concerned in the world, the task to explore new energy and material as a replacement of petroleum is urgent. So it is necessary to harvest and recycle

\footnotetext{
* The research is supported by National High-tech R\&D Program (863 Program) (project number: 2009AA043604).
} 
root stubble for the sake of providing raw material for biomass transformation and utilization.

In the study, Solidworks2009 was applied for the design of rotary root stubble digging machine. With this software, 3 dimensional entity models of each part can be designed and assembled together easily and interference between components can be checked conveniently. Therefore, before manufacture of physical prototype, sufficient assembly and test can be done on simulated models, promoting the standardization, normalization and serialization of the design work.

\section{Characteristics of Solidworks}

Solidworks is the first 3 dimensional CAD software developed on windows operating system, and due to its powerful functions, characteristics of easy to learn and easy to use, it is widely applied in mechanical design. With parametric feature modeling technology, different entities can be created, meeting most requirements of engineering design; with single internal database, all data is related with each other, modifications on dimension in any module will automatically reflect in other modules; in 3 dimensional assembly module, transmission relationship between components can be dynamically simulated. Solidworks is especially suitable for product development, as it is able to shorten product design cycle, improve design quality and reduce cost. Soliworks has become one of the mainstream software in mechanical design and modeling[2].

\section{Design Process of Rotary Root Stubble Digging Machine Using Solidworks}

\subsection{Scheme Design}

The machine consisted of 4 main parts including frame, transmission mechanism, suspension mechanism and digging mechanism. The machine was hitched to tractor with suspension mechanism. During operation, power of tractor was transmitted to input shaft of the machine through universal joint from the tractor's PTO (power take off) shaft. Then via bevel gear pair and bevel-shaft, the power was transmitted to flank chain sprockets. Finally, flank chain sprocket drove the driven sprocket which was fixed on the digging mechanism, so the digging mechanism rotated with the driven sprocket synchronously.

Motion of the digging mechanism was structured by two components. While advancing with tractor (pulled by tractor), it also rotated around the self blade shaft. During working process, root stubble was dug out of soil and tossed upward by the rotating blades, and then the root stubble hit the baffle board and fell behind the machine. After a few days of exposure under the sun, these root stubble were picked up by the matched picking machine which could finally get clean root stubble as there was soil removing mechanism on the machine. In this paper, the task was to dig the root stubble out of soil and the subsequent picking procedure was not discussed here.

The gear box had 1 input shaft and 2 output shafts rotate at opposite directions. To meet different tillage requirements, the digging mechanism can be switched between reverse rotation and forward rotation through manual changes of the transmission 
chain's installation position from left to right. Noted that though there were two pairs of flank sprockets, there was only one strip of chain, so only a pair of flank sprockets (either the right pair or the left pair) was at work at a time. Transmission sketches of reverse rotation and forward rotation were shown in (a) and (b) of fig.1 respectively.

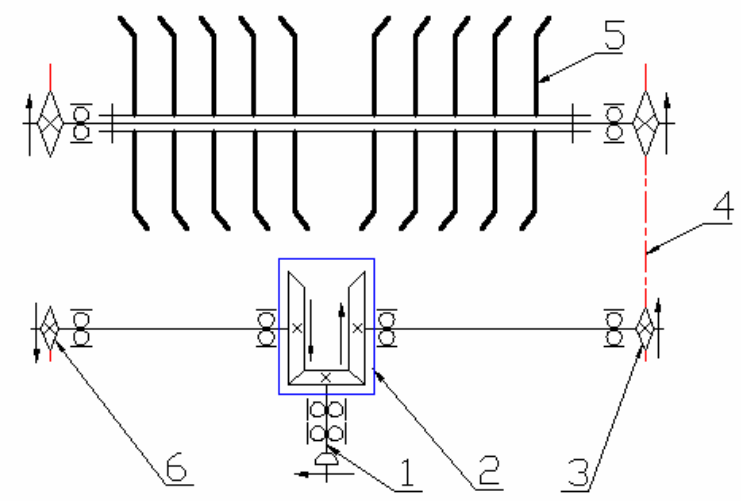

(a)

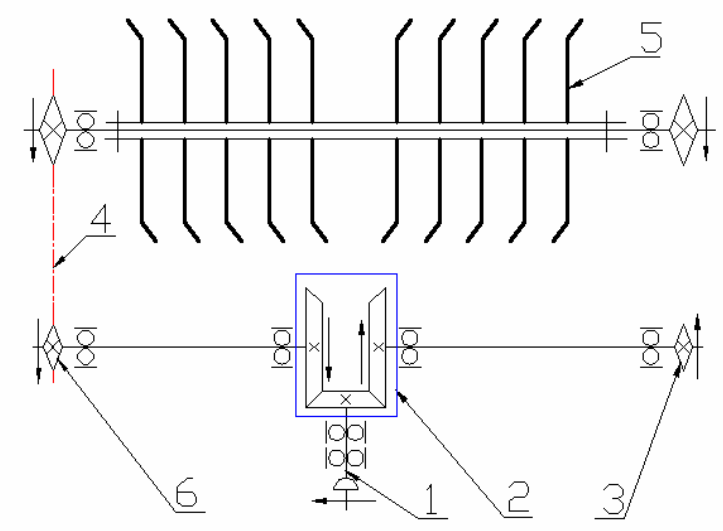

(b)

Fig. 1. Transmission sketches

1. Input shaft; 2. Gear box; 3. Flank sprocket (right);

4. Transmission chain; 5. Digging mechanism; 6. Flank sprocket (left)

\subsection{Part Design ${ }^{[3-6]}$}

Part design is the basis of 3 dimensional virtual design. In Solidworks, features are created from ways such as extrude, revolve, sweep, etc., and then combined together according to constraint relations to form parts. For example, the creating process of the bevel gear used in the machine was: create the basic feature by revolving the 2-D sketch around an axis(revolve) $\rightarrow$ create gear groove by removing material between the two profiles(loft-cut) $\rightarrow$ array gear groove around the axis(circular pattern) $\rightarrow$ create hole and keyway by cutting material(extrude-cut) $\rightarrow$ complete. The process is shown in fig. 2 . 


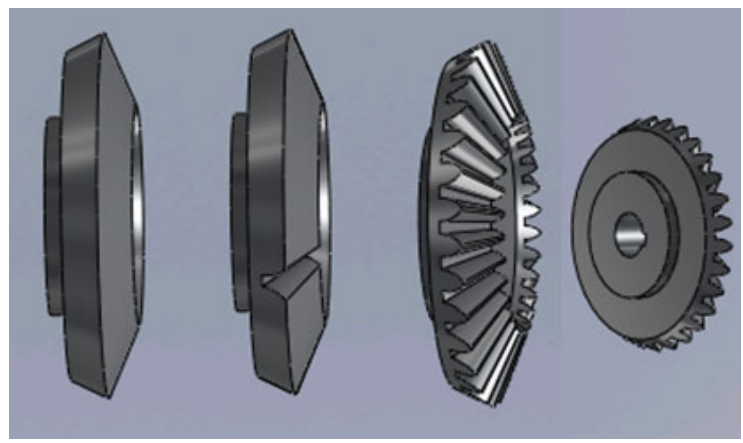

Fig. 2. Creating process of the bevel gear

Parts of the digging machine were created one by one and then saved in the same file folder, as this would make the file management more convenient especially in the subsequent assembly manipulation. During the design process, relationships among features must be taken into account. Generally, according to the order in which features are created, features and their relationships are listed in FeatureManager design tree on the left side of the interface. And for the convenience of feature modification, models can be zoomed in and out, freely rotated, hided and suppressed.

\subsection{Assembly Design}

After parts design was completed, parts (or components) and necessary mates were inserted into assembly environment to form assembly models. Mates create geometric relationships between assembly components and define spatial position of one component relating to another. There are many mate types available in Solidworks such as coincident, parallel, perpendicular, tangent, concentric, and so on. For the machine, according to the function of each mechanism, parts were assembled to 4 subassemblies including frame, transmission mechanism, suspension mechanism and

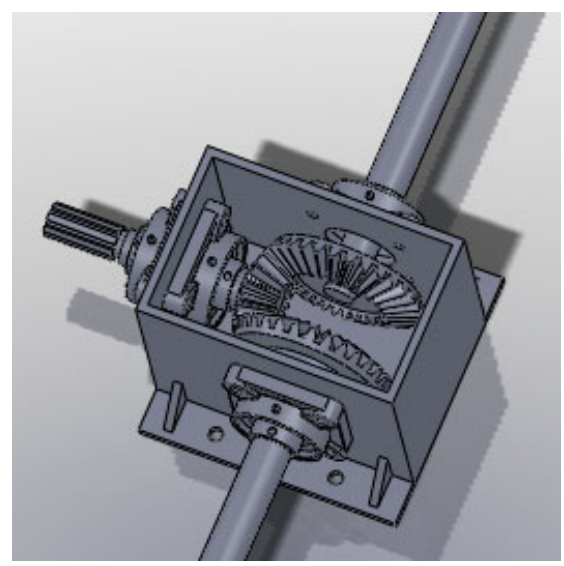

Fig. 3. Assembly of the gear box 
digging mechanism. Then, these 4 sub-assemblies were combined together to form the complete assembly of root stubble digging machine. The gear box and the whole assembly were shown in fig. 3 and fig. 4 respectively.

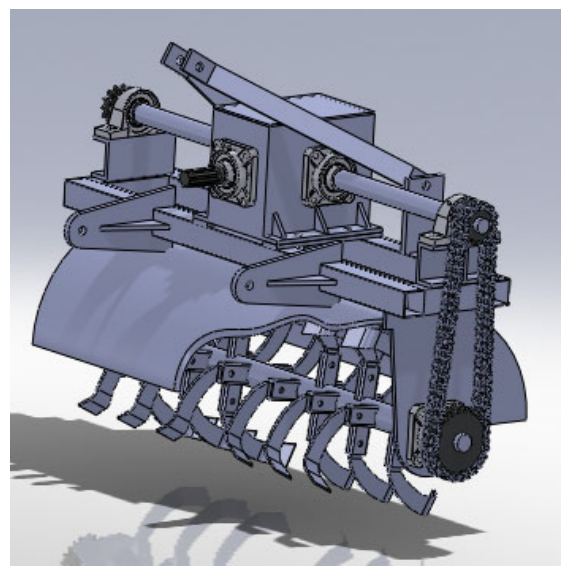

Fig. 4. Whole assembly of the machine

\subsection{Interference Detection}

Interference detection is one of the most important functions of Solidworks which can rapidly determine whether there is any interference between components and between sub-assemblies (a sub-assembly is treated as a single component). Here, the whole assembly was checked for interference, and according to analysis results, relevant details of parts and constraint settings between components were modified. The procedure was repeated until there wasn't any interference, as shown in fig.5.

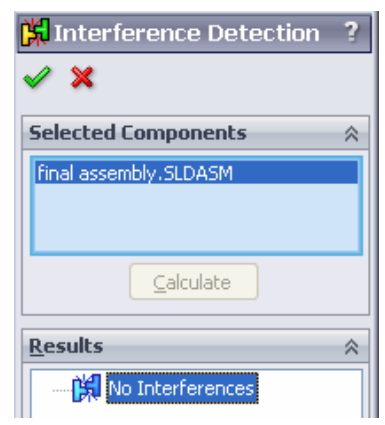

Fig. 5. Interference detection on the machine

\subsection{Generation of 2-D Engineering Drawings}

After above steps, 2-D engineering drawings were generated from corresponding parts and assemblies in the drawing module, and automatic dimensioning was done in 
each drawing. Noted that 3-D models and 2-D engineering drawings were related with each other, namely any modification of dimensions made in 3-D part and assembly module would be reflected in drawing module and vice versa. Some necessary annotations such as weld symbol, geometric tolerance, surface finish symbol and BOM (Bill of Material), etc. were inserted into drawings as these were required for manufacture. Completed engineering drawings were saved in default file format of Solidworks and DWG format which was recognizable by AutoCAD. 2-D projection drawing of the whole machine was shown in fig.6.

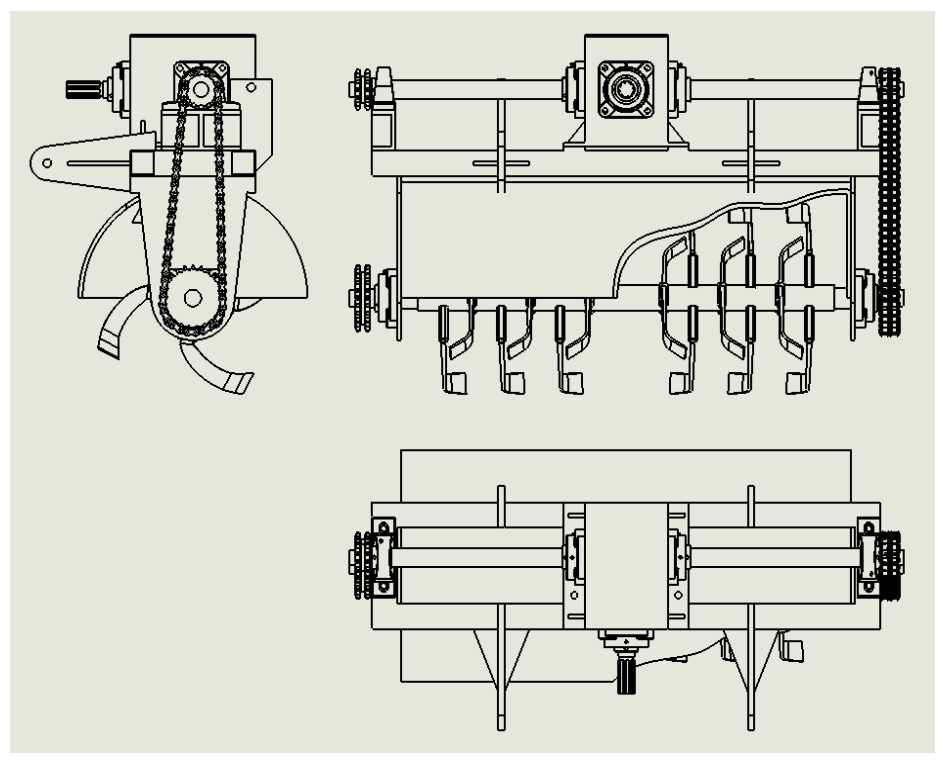

Fig. 6. 2-D projection drawing

\section{Conclusions}

(1) The necessity of mechanized root stubble harvesting and recycling was put forward from the perspective of biomass energy utilization, considering the traditional treating ways and its ingredient of high heating value and low sulfur.

(2) Soliworks was applied to accomplish parts design, assembly design, interference detection and generation of 2-D engineering drawings. Results showed that the design was reasonable and feasible.

(3) The created parts and assembly will be models for subsequent simulation and analysis if necessary. The study provides theoretical foundations and methodological references for the application of virtual prototype technology on the development of new agricultural machinery.

\section{Acknowledgements}

The research is supported by National High-tech R\&D Program (863 Program) (project number: 2009AA043604). 


\section{References}

1. Han, Z.: The Study of Realization Way on Corn Mechanization. Journal, Farm Machinery (05), 45-46 (2010) (in Chinese)

2. Zhou, D., Liu, X., Lu, W.: Application of Solidworks Software on the Design of Agricultural Machinery. Journal, Modernizing Agriculture (10), 42-43 (2006) (in Chinese)

3. Zhu, K., Ning, E., Zhao, M., et al.: Virtual Design and Experiments of 9QS8 Forage Harvester Based on Solidworks. Journal of Agricultural Mechanization Research (11), 137139 (2009)

4. Yu, J., Kong, X., Huang, S., et al.: Virtual Design of Soil-processor in Rice-seedling Raising-by-plates by SolidWorks. Journal, Packaging and Food Machinery 24(2), 31-34 (2006) (in Chinese)

5. Yang, W., Guan, C., Wu, M., et al.: Feature Modeling and Assembling. Conjunction Design on Precision Seeder by Software Solidworks (3), 110-113 (2006) (in Chinese)

6. Zhan, D.: Solidworks Baodian. Publishing House Of Electronics Industry, Beijing (2008) (in Chinese) 